\title{
Structure of Small Platinum Clusters Revised
}

\author{
Szymon Winczewski ${ }^{1}$, Jaroslaw Rybicki ${ }^{1,2,3}$ \\ ${ }^{1}$ Faculty of Technical Physics and Applied Mathematics \\ Gdansk University of Technology \\ Narutowicza 11/12, 80-952 Gdańsk, Poland \\ e-mail: swinczew@mif.pg.gda.pl; ryba@pg.gda.pl \\ ${ }^{2}$ TASK Computer Centre, Gdansk University of Technology \\ Narutowicza 11/12, 80-952 Gdańsk, Poland \\ ${ }^{3}$ Insitute of Mechatronics, Nanotechnology and Vacuum Techniques \\ Koszalin University of Technology, Ractawicka 5-17, 75-620 Koszalin, Poland
}

(Received: 30 March 2011 revised: 24 November 2011 accepted: 25 November 2011; published online: 15 December 2011)

\begin{abstract}
Systematic studies on the structure of platinum clusters consisting of $N=2-15$ atoms were performed using density functional theory. The results show that up to $N=9$ atoms planar structures are as stable as three- dimensional ones. For larger clusters, both distorted and disordered spatial structures are preferred. The global minima of $N=10-$ and 14-atom clusters were found to possess fcc-like structures with significantly higher stability.
\end{abstract}

Key words: nanoparticles, Pt clusters, density functional theory, catalysis, genetic algorithms

\section{INTRODUCTION}

Platinum clusters are one of the most frequently investigated nano-sized systems. As a highly dispersed form of a chemically active element, they offer high intensity of the key catalytic process. It has been proved that platinum nanoparticles are one of the most efficient heterogeneous catalysts for the oxidation of organic acids [1, 2], alkenes [3] and oxygen in acid solution [4]. Moreover, they have also found application as a fotocatalyst in light-induced water splitting on the $\mathrm{TiO}_{2}$ surface $[5,6]$. Since they are considered the most promising materials for fuel cell applications, it is obvious that gaining insight into mechanisms that govern their properties is one of the main and most challenging tasks for nanotechnologists.

The morphology of small platinum clusters has been investigated by several groups at different levels of theory [7-15]. In spite of the overall effort that has been involved in these studies, there is still no agreement as to which structures are preferred. Even with first-principles calculations significant discrepancies were encountered. Yang and co-workers [9] using density functional theory (DFT) in local density approximation (LDA) studied the equilibrium geometries of $N=2-6$-atom clusters. They found that planar structures are strongly preferred, also reporting that the global minimum (GM) of an $N=13$-atom cluster forming an icosahedron distorted by the Jahn-Teller effect. Only one year later, Watari and Ohnishi [10] showed that taking the spin-orbit effect into account reverses the situation, making the decahedral structure distorted by the JahnTeller effect more stable. Also, a different ground state for the $N=13$ atom cluster has been determined in [11].

Recently, Nie and co-workers [14] have investigated the structural evolution of clusters consisting of $N=2-15$ atoms. According to these authors, there is no evident cluster size at which a transition from planar to non-planar arrangement would take place. The presented results are in disagreement with those published by Bhattacharyya and Majumder [15], who instead claim that there is a clear boundary between $N=9$ and $N=10$. A similar claim that two-dimensionality is favored was made in [11], where planar structures were found to be almost as stable as spa- 
tial configurations, and the same transition size was obtained.

Although it is known that the incorporation of spinorbit coupling can be crucial for accurate description of such complex systems as transition metals [16], in most of the literature cited above these were not directly taken into account. We believe this may be the reason for the observed discrepancies. In this work we make an attempt to verify the results that have already been reported.

The main scope of this work is two-fold and can be formulated as a (i) reexamination and (ii) extension of the inconsistent reports found in the literature. We focus on the structure of small platinum clusters consisting of $N=2-15$ atoms. We performed a global search driven by a genetic algorithm, coupled with the semi-empirical Voter-Chen potential used as a first approximation of the energetics. The most stable isomers found in this way were subsequently subjected to local relaxation using DFT. With the intention to achieve (ii), the lowest energy structures reported in $[11,14,15]$ were re-examined. This paper is organized as follows. Section II describes the adopted methodology. This is followed by a discussion of the results. Finally, in Section IV we present conclusions.

\section{METHODOLOGY}

With computations based on higher levels of theory, the possibilities of exploring the configurational space of many-atom systems are significantly limited. Since semiempirical potentials provide a good quality-to-cost ratio, their direct incorporation as a preliminary measure emerges as one of only a few viable approaches. Since this approach has previously been found to be successful (cf. e.g. contributions by Johnston and co-workers [17-21]), we decided to employ it, dividing the task of performing an exhaustive and accurate search for optimal structures into three stages. Firstly, on the basis of a global search carried out under a semi-empirical description numerous candidate structures have been located. Secondly, a small fraction of the initially located configurations (those most stable) was subjected to quantum-based local relaxation. Finally, as it was stated in the introduction, also the lowest energy structures reported in $[11,14,15]$ were verified at the DFT level.

\section{II.1. Voter-Chen potential}

To describe the interactions of platinum atoms, as a first approximation we used the semi-empirical Voter-Chen potential [22], which belongs to the class of embedded atom method (EAM) potentials, where the energy of an $\mathrm{N}$-atom system is written as a sum

$$
\mathrm{E}_{\mathrm{tot}}=\sum_{i=1}^{N} E_{i}
$$

of terms due to individual atoms, i.e.

$$
E_{i}=\frac{1}{2} \sum_{j \neq i}^{N} \phi\left(r_{i j}\right)+F\left(\rho_{i}\right) .
$$

Here, $r_{i j}$ is the distance between atoms $i$ and $j$, the first term accounts for the repulsion of the ion cores, whereas the latter expresses the amount of energy needed to remove atom $i$ from the background electron cloud of density

$$
\rho_{i}=\sum_{j \neq i}^{N} \rho\left(r_{i j}\right) .
$$

Here, $\rho\left(r_{i j}\right)$ is the density due to atom $j$ at the site of atom $i$.

In the case of the Voter-Chen potential, the pair interaction is described by Morse's formula,

$$
\phi\left(r_{i j}\right)=D_{M}\left[1-e^{\alpha_{M}\left(r_{i j}-R_{M}\right)}\right]^{2}-D_{M} .
$$

The electron density term is taken as

$$
\rho\left(r_{i j}\right)=r_{i j}^{6}\left(e^{-\beta r_{i j}}+2^{9} e^{-2 \beta r_{i j}}\right) .
$$

The form of the embedding function $F(\rho)$ was obtained from a fitting procedure under the constraint that the cohesive energy $E_{\text {coh }}$, the equilibrium lattice constant $a_{0}$ and the bulk modulus $B$ be reproduced exactly. The properties of diatomic molecules were also taken into account. This distinguishes the Voter-Chen potential from other EAM-based descriptions and justifies its use as a first approximation of the energetics of nanometer-scale systems.

In Table 1 we present the values of the parameters of the Voter-Chen potential. The values for several properties of $\mathrm{Pt}$, both experimental and calculated using the VoterChen force field, can be found in Table 2.

Table 1. Parameters of the Voter-Chen potential for Pt, after [22]

\begin{tabular}{l|l}
\hline Parameter & Value \\
\hline$D_{M}(\mathrm{eV})$ & 0.76551 \\
\hline$R_{M}(\AA)$ & 2.54446 \\
\hline$\alpha_{M}\left(\AA^{-1}\right)$ & 2.0035 \\
\hline$\beta\left(\AA^{-1}\right)$ & 3.84120 \\
\hline$r_{\text {cutoff }}(\AA)$ & 5.5758 \\
\hline
\end{tabular}


Table 2. Certain properties of platinum. $C_{11}, C_{12}$ and $C_{44}$ - elastic constants, $\Delta E_{\mathrm{vac}}^{f} \quad$ - vacancy formation energy, $D_{e}$ and $R_{e}$ - binding energy and bond length of a diatomic molecule. All data were taken from [22]

\begin{tabular}{l|c|c}
\hline \multicolumn{1}{c|}{ Property } & Experimental & Calculated \\
\hline$E_{\mathrm{coh}}\left(\mathrm{eV} \mathrm{atom}^{-1}\right)$ & 5.77 & 5.77 \\
\hline$a_{0}(\AA)$ & 3.92 & 3.92 \\
\hline$B\left(10^{12} \mathrm{erg} \mathrm{cm}^{-3}\right)$ & 2.83 & 2.83 \\
\hline$C_{11}\left(10^{12} \mathrm{erg} \mathrm{cm}^{-3}\right)$ & 3.21 & 3.47 \\
\hline$C_{12}\left(10^{12} \mathrm{erg} \mathrm{cm}^{-3}\right)$ & 2.64 & 2.51 \\
\hline$C_{44}\left(10^{12} \mathrm{erg} \mathrm{cm}^{-3}\right)$ & 0.78 & 0.74 \\
\hline$\Delta E_{\mathrm{vac}}^{f}(\mathrm{eV})$ & 1.49 & 1.5 \\
\hline$\left.D_{e}(\mathrm{eV} \mathrm{atom})^{-1}\right)$ & 3.15 & 3.17 \\
\hline$R_{e}(\AA)$ & 2.34 & 2.45 \\
\hline
\end{tabular}

\section{II.2. Search technique}

Describing the potential energy surface (PES) of manyatom systems is not a trivial task. Since the number of local minima of energy increases exponentially with the size of the system, it is also difficult to locate only the configurations that are highly stable. Although many search methods have been elaborated, none of them ensures finding the global minimum. For all of them, the probability of success mostly depends on the volume of the solution space that has been searched and the effectiveness of particular techniques is highly related to the considered optimization problem. It is known that in the case of complex configurational search techniques based on genetic algorithms (GAs) can be extremely effective, see e.g. contributions by Johnston et al. [17-21] and other authors [23, 24]. As the Voter-Chen potential generates complicated PESs we decided to employ genetic algorithms as the main search tool.

In so doing we mainly followed the prescriptions given by Johnston in his seminal work [21]. In Figure 1 we present the flowchart of the implemented algorithm. As it is similar to a standard GA we omit a detailed description, presenting only crucial information on the encoding method and on how the particular genetic operators have been realized.

We employed the encoding of Zeiri [24], using realvalued Cartesian coordinates as genes. At the beginning of the search, the initial population (consisting of $N_{\text {pop }}$ specimens) was generated randomly [29]. For every individual, the set of $3 N$ genes (corresponding to the Cartesian coordinates of the atoms of the cluster) was chosen such that the coordinates were located within a sphere of radius $r_{\text {loc }}=3.5 \AA$, which ensured that the density of created clusters was nearly correct.

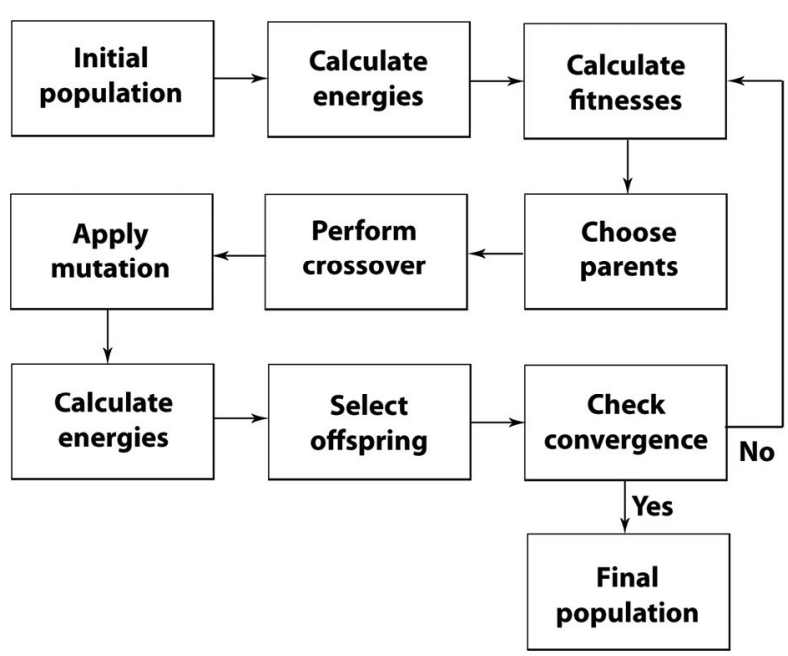

Fig. 1. Flow chart of the implemented genetic algorithm

For a given initial set of individuals, evolution was carried out using the operators of crossover, mutation and selection. Mating was realized with the so-called cut and splice variant (see Fig. 2), proposed by Deaven and Ho [25]. This approach is known to be one of the most effective crossover operators for the optimization of cluster geometries. Mutation was realized by giving a new random value to a randomly selected gene (the constraint keeping the coordinates within a sphere of radius $r_{\text {loc }}$ still applied). Mutation was affecting not only the chromosomes of the offspring, but also those of the parents. The probability $P_{\text {mutate }}$ of undergoing mutation was identical for all specimens. As a result, the implemented GA was operating on a set of $\left(N_{\text {pop }}+N_{\text {child }}\right)\left(1+P_{\text {mutate }}\right)$ candidate configurations (here, $N_{\text {child }}$ denotes the number of offspring).

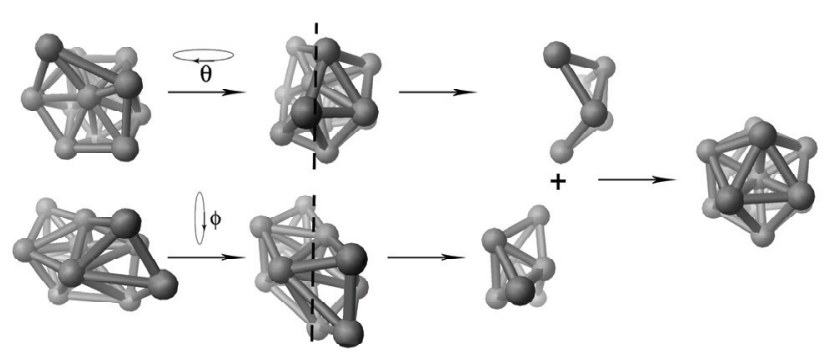

Fig. 2. Schematic representation of the cut and splice mating operator. The geometries of parents are initially rotated by random angles. Then a cutting plane is chosen (also at random) and the offspring is produced by splicing the two resulting parts 
The weighing of the quality of particular individuals was accomplished by using dynamic fitness scaling, i.e. the fitness of $i$-th specimen was determined from

$$
f_{i}=f\left(\varepsilon_{i}\right)=f\left(\frac{E_{i}-E_{\min }}{E_{\max }-E_{\min }}\right) .
$$

In the above, $E_{\mathrm{i}}$ and $\varepsilon_{\mathrm{i}}$ denote the energy and reduced energy of the $i$-th individual, respectively, $E_{\max }$ and $E_{\min }$ stand for the maximum and minimum value of $E_{\mathrm{i}}$, respectively, in the entire population, and $f$ is the fitness function. Proportionate selection was used, where the probability of having the $i$-th individual in the parental pool is directly proportional to its fitness $f_{i}$. Subsequent generations were formed by natural selection - as a set of individuals with the lowest values of energy. Evolution was regarded as converged if no change in $E_{\min }$ was observed for $N_{\text {conv }}$ successive iterations.

At the beginning of the calculations we have performed a number of numerical experiments in order to determine the optimal set of the parameters and the most suitable form of the fitness function. Statistical considerations led to $f(\varepsilon)=$ $\exp (-2.5 \varepsilon), N_{\text {pop }}=20, N_{\text {child }}=18, P_{\text {mutate }}=0.1$ and $N_{\text {conv }}=10$. These values were used in all subsequent searches.

A global search employing the algorithm described above was performed for $N=3-15$-atom clusters. The main goal at this stage was obtaining numerous low-lying configurations. For every atomization $N$ we performed up to 20000 distinct searches. Simple evaluation allows us to claim that the adopted GA has analyzed in a comparative manner up to $4 \times 10^{7}$ distinct geometries for every cluster size. As a result, about four thousand distinct stable isomers were localized during an exhaustive global search.

\section{II.3. Details of the first-principles calculations}

The results of semi-empirical calculations were subsequently verified at the DFT level of theory. A certain number of previously located isomers (namely, up to six lowest-lying arrangements for every atomization $N$ ) was subjected to further local relaxation. Also, the highly stable structures reported in $[11,14,15]$ were re-examined. It should be noted that the GA was successful in finding most of these (or very similar) structures, which further demonstrates the exhaustiveness of the employed global search. To underline the effort involved in this study, we note that 144 configurations were optimized under sophisticated quantum treatment.

All of the quantum-based computations were carried out using the Gaussian program [26] in a spin unrestricted scheme. Zero-temperature DFT under the generalized gradient approximation was used, employing the PerdewWang 91 exchange-correlation functional [27, 28]. We stress that the PW91 functional (in contrast to the hybrid functionals, which are nowadays often used), does not employ any empirical parameters. With the intention to express the Kohn-Sham eigenlevels, the (7s6p5d)/[6s3p2d] basis set of Ahlrichs et al. was adopted [30]. The presence of core electrons was taken into account by the use of an effective core potential (ECP) derived by Andrae and coworkers [31]. We note that the adopted ECP accounts for relativistic spin-orbit effects, which are crucial for an accurate description of dense, open-shell systems.

Local quantum relaxation was performed without imposing any symmetry constraints. This procedure, although increasing the computational complexity, allowed us to locate distorted and even disordered structures. Strict convergence criteria were always used during optimization, the total energies and bond lengths were converged to $10^{-3} \mathrm{eV}$ and $10^{-3} \AA$, respectively. Due to the applied UHF scheme, the relaxation was also carried out in the total spin domain. After beginning with a singlet state, the minimization was successively renewed for higher multiplet states. The process was continued until there was no drop in the total energy observed for two consecutively higher spin states. We note that it was often difficult to achieve self-consistency by using the computationally fast DIIS method. Consequently, only the quadratically-convergent SCF method was used, which increased the overall calculational effort.

\section{RESULTS}

The calculated equilibrium structures are summarized in Tables 3-8. For the sake of clarity, we report only 83 from the 131 identified arrangements (showing those with lowest energies or otherwise particularly important). For every isomer we give its schematic representation (figures were obtained with MacMolPlt program [32]) and its notation by using a $\mathrm{Pt}_{N}^{X}$ pattern. Here, $N$ denotes the cluster size, whereas $X$ is used to label isomers of the same size. In order to analyze the energetics and relative stability of the investigated clusters, we calculated the binding energy, which is defined as

$$
E_{b}\left(\mathrm{Pt}_{N}\right)=E_{\text {tot }}\left(\mathrm{Pt}_{1}\right)-\frac{E_{\mathrm{tot}}\left(\mathrm{Pt}_{N}\right)}{N} \text {. }
$$

In the above $E_{\text {tot }}\left(\mathrm{Pt}_{1}\right)$ and $E_{\text {tot }}\left(\mathrm{Pt}_{N}\right)$ denote the total potential energies (calculated at the DFT level) of a single atom and an $N$-atom cluster, respectively. In this work $E_{b}$ is expressed in $\mathrm{eV}$ per atom and we give its value only for global minima (GM). For other (non-global) configurations, 
Table 3. Lowest energy structures of $N=2-4$ atom clusters

\begin{tabular}{|c|c|c|c|c|c|}
\hline Notation & $\mathrm{Pt}_{2}^{\mathrm{A}}$ & $\mathrm{Pt}_{3}^{\mathrm{A}}$ & $\mathrm{Pt}_{3}^{\mathrm{B}}$ & $\mathrm{Pt}_{3}^{\mathrm{C}}$ & $\mathrm{Pt}{ }_{3}^{\mathrm{D}}$ \\
\hline Geometry & & & & $0-0$ & \\
\hline$E_{b}(\mathrm{eV} /$ atom $)$ & 1.655 & 2.232 & 0.030 & 0.225 & 0.267 \\
\hline$\mu\left(\mu_{B} /\right.$ atom $)$ & 0.50 & 0.33 & 0 & 0.67 & 0.33 \\
\hline Bond length $(\AA)$ & 2.40 & 2.55 & $2.53-2.54$ & 2.42 & 2.39 \\
\hline Notation & $\mathrm{Pt}_{4}^{\mathrm{A}}$ & $\mathrm{Pt}_{4}^{\mathrm{B}}$ & $\mathrm{Pt}_{4} \mathrm{C}$ & $\mathrm{Pt}_{4}^{\mathrm{D}}$ & $\mathrm{Pt}_{4}^{\mathrm{E}}$ \\
\hline Geometry & & & & & $0-0-0$ \\
\hline$E_{b}(\mathrm{eV} /$ atom $)$ & 2.489 & 0.028 & 0.048 & 0.140 & 0.542 \\
\hline$\mu\left(\mu_{B} /\right.$ atom $)$ & 0.25 & 0.25 & 0.25 & 0.25 & 0.50 \\
\hline Bond length $(\AA)$ & $2.62-2.73$ & $2.56-2.65$ & 2.52 & $2.39-2.55$ & $2.42-2.52$ \\
\hline
\end{tabular}

Table 4. Lowest energy structures of $N=5$ atom clusters

\begin{tabular}{|c|c|c|c|c|c|c|}
\hline Notation & $\mathrm{Pt}_{5}^{\mathrm{A}}$ & $\mathrm{Pt}_{5}^{\mathrm{B}}$ & $\mathrm{Pt}_{5}^{\mathrm{C}}$ & $\mathrm{Pt}_{5}^{\mathrm{D}}$ & $\mathrm{Pt}_{5}^{\mathrm{E}}$ & $\mathrm{Pt}_{5}^{\mathrm{F}}$ \\
\hline Geometry & & & & & & \\
\hline$E_{b}(\mathrm{eV} /$ atom $)$ & 2.677 & $4 \cdot 10^{-4}$ & 0.005 & 0.024 & 0.036 & 0.093 \\
\hline$\mu\left(\mu_{B} /\right.$ atom $)$ & $\begin{array}{c}0.40 \\
263-270\end{array}$ & $\begin{array}{c}0.40 \\
255-2.94\end{array}$ & $\begin{array}{c}0.40 \\
255-267\end{array}$ & $\begin{array}{c}0.40 \\
262-271\end{array}$ & $\begin{array}{c}0.20 \\
245-2.59\end{array}$ & $\begin{array}{c}0.20 \\
250-256\end{array}$ \\
\hline
\end{tabular}

Table 5. Lowest energy structures of $N=6$ atom clusters

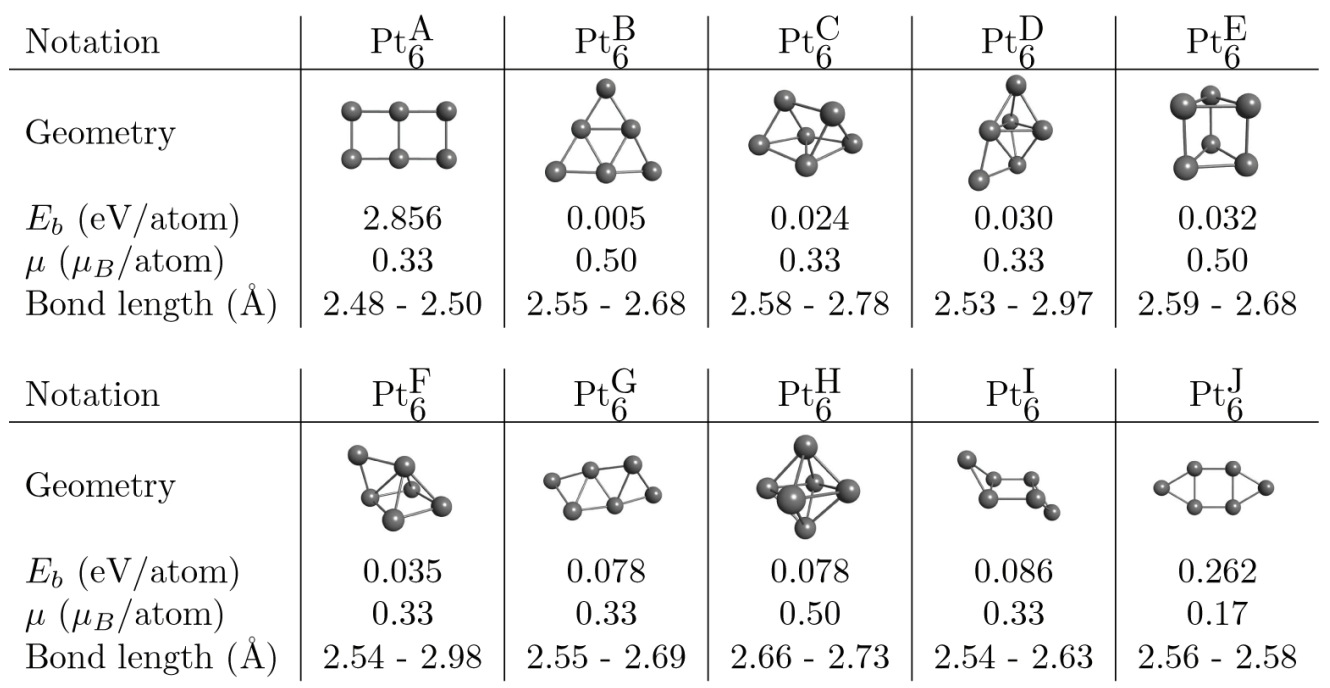


Table 6. Lowest energy structures of $N=7-9$ atom clusters

\begin{tabular}{|c|c|c|c|c|c|}
\hline Notation & $\mathrm{Pt}_{7}^{\mathrm{A}}$ & $\mathrm{Pt} \frac{\mathrm{B}}{7}$ & $\mathrm{Pt}_{7}^{\mathrm{C}}$ & $\mathrm{Pt} \frac{\mathrm{D}}{7}$ & $\mathrm{Pt} \frac{\mathrm{E}}{7}$ \\
\hline Geometry & & & & & \\
\hline$E_{b}(\mathrm{eV} /$ atom $)$ & 2.975 & 0.003 & 0.014 & 0.027 & 0.046 \\
\hline $\begin{array}{l}\mu\left(\mu_{B} / \text { atom }\right) \\
\text { Bond length }(\AA)\end{array}$ & $\begin{array}{c}0.43 \\
2.59-2.98\end{array}$ & $\begin{array}{c}0.43 \\
2.53-3.01\end{array}$ & $\begin{array}{c}0.14 \\
2.56-2.69\end{array}$ & $\begin{array}{c}0.29 \\
2.50-2.80\end{array}$ & $\begin{array}{c}0.29 \\
2.62-2.86\end{array}$ \\
\hline
\end{tabular}

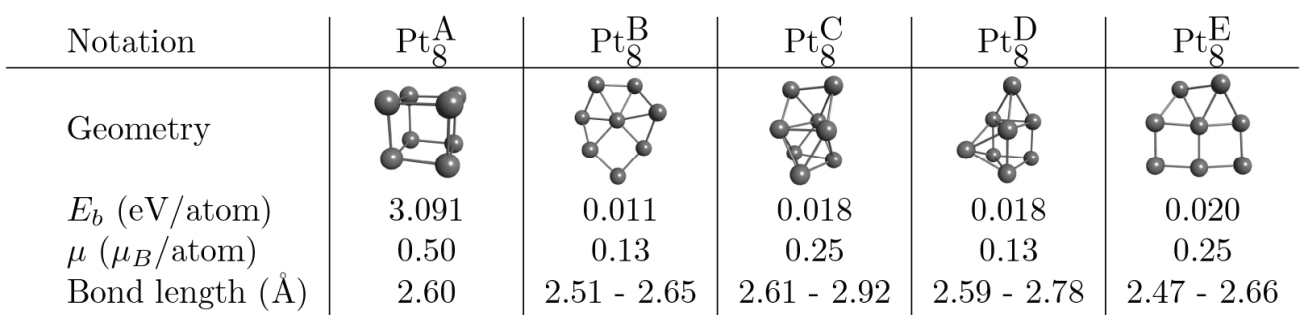

\begin{tabular}{|c|c|c|c|c|c|}
\hline Notation & $\mathrm{Pt}_{9}^{\mathrm{A}}$ & $\mathrm{Pt}_{9}^{\mathrm{B}}$ & $\mathrm{Pt}_{9}^{\mathrm{C}}$ & $\mathrm{Pt}_{9}^{\mathrm{D}}$ & $\mathrm{Pt}_{9}^{\mathrm{E}}$ \\
\hline Geometry & 8 & & & & \\
\hline $\begin{array}{l}E_{b}(\mathrm{eV} / \text { atom }) \\
\mu\left(\mu_{B} / \text { atom }\right) \\
\text { Bond length }(\AA)\end{array}$ & $\begin{array}{c}3.236 \\
0.33 \\
2.57-2.85\end{array}$ & $\begin{array}{c}0.020 \\
0.44 \\
2.60-2.90\end{array}$ & $\begin{array}{c}0.033 \\
0.22 \\
2.59-2.79\end{array}$ & $\begin{array}{c}0.036 \\
0.00 \\
2.59-3.09\end{array}$ & $\begin{array}{c}0.053 \\
0.11 \\
2.55-2.80\end{array}$ \\
\hline
\end{tabular}

Table 7. Lowest energy structures of $N=10-12$ atom clusters

\begin{tabular}{|c|c|c|c|c|c|c|c|}
\hline Notation & $\mathrm{Pt}_{10}^{\mathrm{A}}$ & $\mathrm{Pt}_{10}^{\mathrm{B}}$ & $\mathrm{Pt}_{10}^{\mathrm{C}}$ & $\mathrm{Pt}_{10}^{\mathrm{D}}$ & $\mathrm{Pt}_{10}^{\mathrm{E}}$ & $\mathrm{Pt}_{10}^{\mathrm{F}}$ & $\mathrm{Pt}_{10}^{\mathrm{G}}$ \\
\hline Geometry & 20 & & op & \&ी & & & \\
\hline $\begin{array}{l}E_{b}(\mathrm{eV} / \text { atom }) \\
\mu\left(\mu_{B} / \text { atom }\right) \\
\text { Bond length }(\AA)\end{array}$ & $\begin{array}{c}3.384 \\
0.40 \\
2.60-2.83\end{array}$ & $\begin{array}{c}0.105 \\
0.10 \\
2.57-2.68\end{array}$ & $\begin{array}{c}0.108 \\
0.20 \\
2.53-2.78\end{array}$ & $\begin{array}{c}0.115 \\
0.30 \\
2.58-2.90\end{array}$ & $\begin{array}{c}0.116 \\
0.00 \\
2.56-3.08\end{array}$ & $\begin{array}{c}0.118 \\
0.00 \\
2.56-2.65\end{array}$ & $\begin{array}{c}0.127 \\
0.20 \\
2.60-3.04\end{array}$ \\
\hline
\end{tabular}

\begin{tabular}{|c|c|c|c|c|c|c|c|}
\hline Notation & $\mathrm{Pt}_{11}^{\mathrm{A}}$ & $\mathrm{Pt}_{11}^{\mathrm{B}}$ & $\mathrm{Pt}_{11}^{\mathrm{C}}$ & $\mathrm{Pt}_{11}^{\mathrm{D}}$ & $\mathrm{Pt}_{11}^{\mathrm{E}}$ & $\mathrm{Pt}_{11}^{\mathrm{F}}$ & $\mathrm{Pt}_{11}^{\mathrm{G}}$ \\
\hline Geometry & & & & & & & \\
\hline $\begin{array}{l}E_{b}(\mathrm{eV} / \text { atom }) \\
\mu\left(\mu_{B} / \text { atom }\right) \\
\text { Bond length }(\AA)\end{array}$ & $\begin{array}{c}3.387 \\
0.00 \\
2.55-2.67\end{array}$ & $\begin{array}{c}0.008 \\
0.36 \\
2.57-2.82\end{array}$ & $\begin{array}{c}0.012 \\
0.27 \\
2.56-3.00\end{array}$ & $\begin{array}{c}0.030 \\
0.00 \\
2.53-2.91\end{array}$ & $\begin{array}{c}0.031 \\
0.18 \\
2.56-2.86\end{array}$ & $\begin{array}{c}0.053 \\
0.18 \\
2.57-2.93\end{array}$ & $\begin{array}{c}0.060 \\
0.18 \\
2.54-2.94\end{array}$ \\
\hline
\end{tabular}

\begin{tabular}{|c|c|c|c|c|c|c|c|}
\hline Notation & $\mathrm{Pt}_{12}^{\mathrm{A}}$ & $\mathrm{Pt}_{12}^{\mathrm{B}}$ & $\mathrm{Pt}_{12}^{\mathrm{C}}$ & $\mathrm{Pt}_{12}^{\mathrm{D}}$ & $\mathrm{Pt}_{12}^{\mathrm{E}}$ & $\mathrm{Pt}_{12}^{\mathrm{F}}$ & $\mathrm{Pt}_{12}^{\mathrm{G}}$ \\
\hline Geometry & & & & 8 & & & \\
\hline $\begin{array}{l}E_{b}(\mathrm{eV} / \text { atom }) \\
\mu\left(\mu_{B} / \text { atom }\right) \\
\text { Bond length }(\AA)\end{array}$ & $\begin{array}{c}3.446 \\
0.17 \\
2.56-3.05\end{array}$ & $\begin{array}{c}0.021 \\
0.17 \\
2.57-3.07\end{array}$ & $\begin{array}{c}0.024 \\
0.17 \\
2.56-2.83\end{array}$ & $\begin{array}{c}0.038 \\
0.17 \\
2.53-2.96\end{array}$ & $\begin{array}{c}0.042 \\
0.00 \\
2.56-3.05\end{array}$ & $\begin{array}{c}0.045 \\
0.00 \\
2.55-2.76\end{array}$ & $\begin{array}{c}0.082 \\
0.08 \\
2.54-2.63\end{array}$ \\
\hline
\end{tabular}


Table 8. Lowest energy structures of $N=13-15$ atom clusters

\begin{tabular}{|c|c|c|c|c|c|c|c|}
\hline Notation & $\mathrm{Pt}_{13}^{\mathrm{A}}$ & $\mathrm{Pt}_{13}^{\mathrm{B}}$ & $\mathrm{Pt}_{13}^{\mathrm{C}}$ & $\mathrm{Pt}_{13}^{\mathrm{D}}$ & $\mathrm{Pt}_{13}^{\mathrm{E}}$ & $\mathrm{Pt}_{13}^{\mathrm{F}}$ & $\mathrm{Pt}_{13}^{\mathrm{G}}$ \\
\hline \multicolumn{8}{|l|}{ Geometry } \\
\hline$E_{b}(\mathrm{eV} /$ atom $)$ & 3.490 & 0.001 & 0.007 & 0.054 & 0.056 & 0.057 & 0.210 \\
\hline$\mu\left(\mu_{B} /\right.$ atom $)$ & 0.08 & 0.15 & 0.00 & 0.15 & 0.00 & 0.15 & 0.23 \\
\hline Bond length $(\AA)$ & $2.55-2.77$ & $2.58-3.06$ & $2.58-2.67$ & $2.56-2.92$ & $2.54-2.80$ & $2.55-3.07$ & $2.64-2.96$ \\
\hline Notation & $\mathrm{Pt}_{14}^{\mathrm{A}}$ & $\mathrm{Pt}_{14}^{\mathrm{B}}$ & $\mathrm{Pt}_{14}^{\mathrm{C}}$ & $\mathrm{Pt}_{14}^{\mathrm{D}}$ & $\mathrm{Pt}_{14}^{\mathrm{E}}$ & $\mathrm{Pt}_{14}^{\mathrm{F}}$ & \\
\hline \multicolumn{8}{|l|}{ Geometry } \\
\hline$E_{b}(\mathrm{eV} /$ atom $)$ & 3.574 & 0.069 & 0.072 & 0.122 & 0.158 & 0.161 & 0.171 \\
\hline$\mu\left(\mu_{B} /\right.$ atom $)$ & 0.14 & 0.29 & 0.07 & 0.07 & 0.00 & 0.07 & 0.21 \\
\hline Bond length $(\AA)$ & $2.61-3.03$ & $2.56-3.02$ & $2.56-2.91$ & $2.58-2.93$ & $2.59-2.94$ & $2.66-2.93$ & $2.54-3.02$ \\
\hline Notation & $\mathrm{Pt}_{15}^{\mathrm{A}}$ & $\mathrm{Pt}_{15}^{\mathrm{B}}$ & $\mathrm{Pt}_{15}^{\mathrm{C}}$ & $\mathrm{Pt}_{15}^{\mathrm{D}}$ & $\mathrm{Pt}_{15}^{\mathrm{E}}$ & $\mathrm{Pt}_{15}^{\mathrm{F}}$ & $\mathrm{Pt}_{15}^{\mathrm{G}}$ \\
\hline \multicolumn{8}{|l|}{ Geometry } \\
\hline$E_{b}(\mathrm{eV} /$ atom $)$ & 3.572 & 0.080 & 0.086 & 0.086 & 0.111 & 0.119 & 0.185 \\
\hline$\mu\left(\mu_{B} /\right.$ atom $)$ & 0.13 & 0.13 & 0.13 & 0.00 & 0.13 & 0.07 & 0.13 \\
\hline Bond length $(\AA)$ & $2.60-3.07$ & $2.64-2.98$ & $2.59-3.09$ & $2.63-2.97$ & $2.63-3.09$ & $2.59-3.09$ & $2.56-3.08$ \\
\hline
\end{tabular}

tions, the energy difference to the corresponding GM is given. The magnetic moments $\mu$ (in Bohr magneton per atom) and the range of bond lengths are also given.

In order to test the reliability of our approach, we first performed calculations for a platinum atom and a dimer. The platinum atom was found to possess a triplet ground state. Its ionization potential and electron affinity were estimated at $9.432 \mathrm{eV}$ and $1.943 \mathrm{eV}$, respectively, which is in satisfactory agreement with the experimentally measured $8.958 \mathrm{eV}$ [33] and $2.123 \mathrm{eV}$ [34], respectively. In the case of the platinum dimer, the triplet ground state had a bond length of $R_{e}=2.396 \AA$ and a binding energy $E_{b}=1.655 \mathrm{eV}$. The stretching frequency of $\mathrm{Pt}_{2}$ was calculated as $\omega=220 \mathrm{~cm}^{-1}$. The obtained results are in close agreement with the available experimental data. Airola and Morse [35] reported $R_{e}=2.332 \AA, E_{b}=1.57 \mathrm{eV}$ and $\omega=222 \mathrm{~cm}^{-1}$, whereas Grushow and Ervin [36] found $R_{e}=2.333 \AA$ and $E_{b}=1.57 \mathrm{eV}$. We note that both in [11] and [15] the agreement was not as good. Although the agreement for the bond length was as good as in this work (Xiao and Wang reported $R_{e}=2.34$, Bhattacharyya and Majumder found $R_{e}=2.33$ ), the calculated values of $E_{b}$ were severely overestimated (1.76 eV and $1.86 \mathrm{eV}$, respectively).

\section{III.1. Structure of $\mathbf{P t}_{3}-\mathbf{P t}_{\mathbf{6}}$ clusters}

The lowest energy structures of $N=3$-6-atom clusters are presented in Tables 3-5. In the case of the platinum trimer four distinct isomers were located. The most stable one has the geometry of an equilateral triangle with a bond length of $2.546 \AA$, a binding energy of $E_{b}=2.231 \mathrm{eV}$ and triplet spin multiplicity. Its excited singlet state is found to lie only $0.006 \mathrm{eV}$ above it, which indicates rather high excitability. The second structure, with a geometry of an isosceles triangle is found at $E_{b}=2.202 \mathrm{eV}$ with a singlet ground state. The linear $\mathrm{Pt}_{3}$ structure was located at $E_{b}=$ $2.006 \mathrm{eV}$, possessing a pentet ground state and a bond length of $2.420 \AA$. It is worth noting that a triatomic chain indicates increased stability of electronic structure. The energy distance to its excited triplet state is found to be significant and equal to $0.223 \mathrm{eV}$. One nearly-linear arrangement was also located.

In the case of the $N=4$-atom cluster, the global minimum is found to be a tetrahedron with a triplet spin multiplicity. The binding energy of the second isomer, a bent rhombus, is found to be lower by $0.028 \mathrm{eV}$. Planar arrangements were also found. The slightly rhombic and linear chain structures were found to lie $0.048 \mathrm{eV}$ and $0.542 \mathrm{eV}$ above the global minimum, respectively. The relatively small stability of the latter (together with the low binding energy of $\mathrm{Pt}_{3}^{C}$ ) indicates that chain structures are not generally favored.

The results obtained for $\mathrm{Pt}_{3}$ differ in many aspects from those reported in $[11,14,15]$. Nevertheless, considerable similarity between our picture and that presented by 
Sebetci in [16] was obtained in the case of the $N=4$-atom cluster. In each case the GM of the four-atom cluster was found to be a tetrahedron, the remaining $N=4$-atom isomers were also predicted similarly. Sebetci found that the second highly stable arrangement has a rhombic shape (equivalent to our $\mathrm{Pt}_{4}^{B}$ ) with a binding energy lower by $0.032 \mathrm{eV}$ with respect to the GM, which is very close to the value of $0.028 \mathrm{eV}$ obtained here. The third lowest-lying structure was recognized by Sebetci as a square (with a binding energy of $0.089 \mathrm{eV}$ below that of the GM). This structure corresponds to our $\mathrm{Pt}_{4}^{C}$. In this case our methodology predicts slightly higher stability, i.e. the $E_{b}$ of $\mathrm{Pt}_{4}^{C}$ is by $0.048 \mathrm{eV}$ lower than for $\mathrm{Pt}_{4}^{A}$. The fourth isomer was also similarly predicted to possess a Y-like shape.

For $\mathrm{Pt}_{5}$, the lowest-lying isomer was found to be a trigonal bipyramid, with a binding energy of $2.677 \mathrm{eV}$, almost isoenergetic with the second isomer in the shape of an edge capped-tetrahedron. A third isomer was found only $0.005 \mathrm{eV}$ above the GM, adopting a W-shaped nearlyplanar geometry.

We note that the three most stable geometries found for $\mathrm{Pt}_{5}$ can be obtained by capping structures found for $\mathrm{Pt}_{4}$. Since there is no change in the relative order of motifs, it is clearly seen that structural trend continues when going from $N=4$ to $N=5$. Our results are in good agreement with [16]. Although a different GM was predicted (Sebetci reported a pyramidal geometry), the ordering of other isomers was predicted similarly.

The first departure from the trend is observed for $N=6$, for which the two lowest-lying isomers were found to be planar. The most stable configuration was a planar doublesquare structure with a binding energy of $2.857 \mathrm{eV}$ and pentet multiplicity. The second was found to possess a nearly-planar triangular geometry with a binding energy higher by only $0.005 \mathrm{eV}$. Since the first three-dimensional configuration was found to lie $0.024 \mathrm{eV}$ above the GM, it is clearly seen that for $N=6$ planar geometries are strongly preferred. This is in contrast to the results of Sebetci, who reports a GM with a trigonal prism shape. On the other hand, Sebetci reports triangular and double square structures to be highly stable, which is in agreement with our finding.

\section{III.2. Structure of $\mathbf{P t}_{7}-\mathrm{Pt}_{15}$ clusters}

Since the distinction between motifs preferred by larger clusters cannot be clearly made (the mixing of different building blocks is strongly preferred) we omit detailed discussion of all geometries located for $N=7-15$-atom clusters. Nevertheless, qualitative notes on regularities that have been found will be presented.

Our results show that above $N=6$ size there is no global minimum which has a planar geometry. However, the relatively high stability of the $\mathrm{Pt}_{7}^{B}$ isomer (which can be seen as $\mathrm{Pt}_{6}^{B}$ with an adatom and thus considered as a nearly-planar rather than a three-dimensional structure) and also of $\mathrm{Pt}_{7}^{D}, \mathrm{Pt}_{8}^{B}$ and $\mathrm{Pt}_{8}^{E}$ isomers shows that the tendency to prefer planarity remains sustained also for $N=7$ and $N=8$. In order to illustrate the observed trends, Figure 3 shows the binding energies of the most stable structures. The additional distinction between clusters of different dimensionality and also (in the case of spatial configurations) of different degree of order was made. It is clearly seen that up to $N=8$ planar (or nearly-planar) structures are as stable as three-dimensional ones. Starting with $N=9$, a deviation from this trend is observed and the most stable planar arrangement of an $N=10$-atom cluster is less stable than the most stable (and even the second most stable) spatial configuration of a nine-atom cluster. This clearly shows that the growth of planar arrangements stops near $N=9$. The same conclusion was drawn by Xiao and Wang [11] and also by Bhattacharyya and Majumder [15].

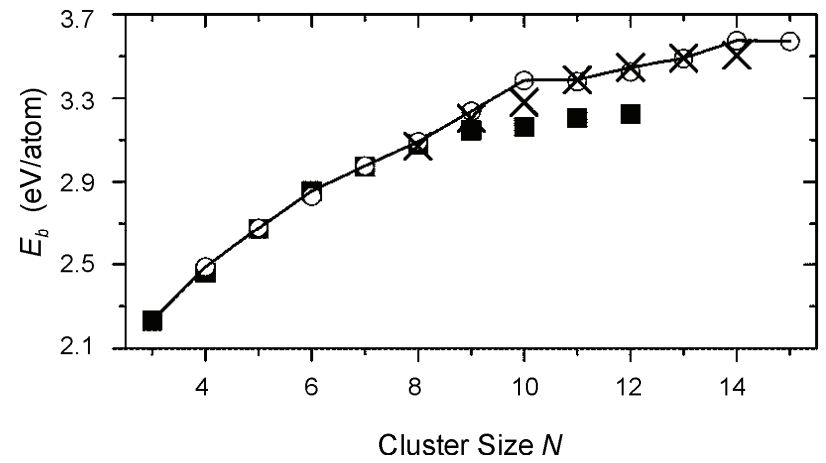

Fig. 3. Binding energy as a function of cluster size for the most stable: (a) planar or nearly-planar (filled squares), (b) spatial, regular or slightly distorted (circles) and (c) spatial, amorphouslike (crosses) structures. The solid line denotes the binding energy of located global minima

The most stable arrangement of an $N=8$ atom cluster forms a cube, whereas $\mathrm{Pt}_{9}$ prefers the geometry of a defected tetrahedron. By adding the lacking atom to its base, the GM of an $N=10$-atom cluster, i.e. a subtly distorted tetrahedron, is formed. The most stable isomer of an $N=11$-atom cluster was found to be highly asymmetric. Since it is composed of many different base building blocks of considerably different nearest neighbor distances, it can be regarded as an amorphous one. Figure 4 shows 
pair correlation functions $g(r)$ of lowest energy structures located for $N=11,12$ and 13. In order to enable a direct comparison, we also plot $g(r)$ for the $\mathrm{Pt}_{14}^{A}$ isomer, which was found to possess a highly ordered fcc-like structure. From the splitting of the second peak of $g(r)$, it is clearly seen that both $\mathrm{Pt}_{12}^{A}$ and $\mathrm{Pt}_{13}^{A}$, are also strongly disordered, similarly to $\mathrm{Pt}_{11}^{A}$. In general, the tendency to prefer amorphous geometries was found to be very strong. About forty percent of all structures located for $N=10$-15-atom clusters were found to be disordered. We note that the above estimate was based not only on the analysis of pair correlation functions. The symmetry of shape was also taken into account in order to distinguish disordered structures from those that are only distorted. It should be noted that amorphous structures indicate binding energies which are comparable to binding energies of highly regular arrangements (see Fig. 3). This indicates that the tendency to prefer regular and nearly-planar structures turns into a tendency to favor regular and amorphous structures beyond $N=9$.
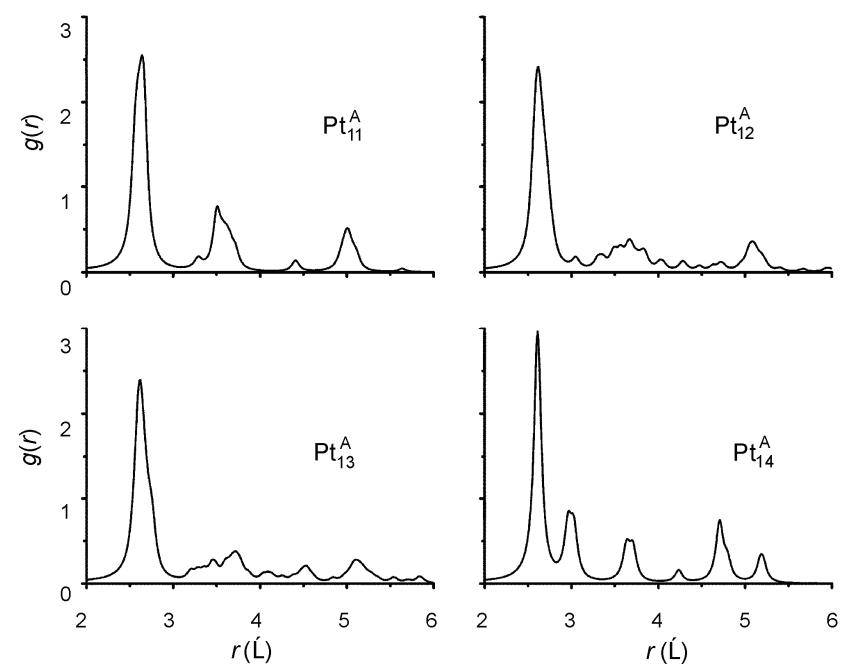

Fig. 4. Pair correlation functions of selected isomers, obtained with a Lorentzian broadening of $0.1 \AA$

Starting with $N=10$, the clusters tend to form fcc-like structures. All of $\mathrm{Pt}_{10}^{A}, \mathrm{Pt}_{10}^{G}, \mathrm{Pt}_{11}^{B}, \mathrm{Pt}_{12}^{B}$ and $\mathrm{Pt}_{13}^{B}$ can be seen as precursors to the $\mathrm{Pt}_{14}^{A}$ cluster. This continues for the $N=15$-atom cluster, whose GM was found to be a capped $\mathrm{Pt}_{14}^{A}$ isomer. The geometry of the $N=13$-atom cluster constitutes an important issue. Our studies show that the structure of an icosahedron (i.e. $\mathrm{Pt}_{13}^{G}$ ) is relatively unstable, with a binding energy lower by as much as $0.21 \mathrm{eV}$ compared to the binding energy of the corresponding amorphous-like global minimum. In general, isomers that were determined by others to be global minima (for example, the cubo-octahedron structure of $\mathrm{Pt}_{13}^{E}$ reported in [10] and [15]) are here predicted to have poor stability.

Many of the $N=10$-14-atom isomers possess a layered structure (e.g. $\mathrm{Pt}_{10}^{D}, \mathrm{Pt}_{11}^{F}, \mathrm{Pt}_{11}^{G}, \mathrm{Pt}_{13}^{C}$ and $\mathrm{Pt}_{14}^{E}$ ), having two dimensions considerably larger than the third. Above $N=13$ the tendency to assume a spherical shape becomes apparent and structures with a high mean coordination number are preferred. Their geometries cannot be clearly characterized, indicating rather high complexity.

\section{III.3. Energetics}

The binding energy of global minima increases smoothly as a function of cluster size (see Fig. 3). However, some anomalies are found for $N=10,14$ showing that these clusters display enhanced stability. In order to illustrate this more clearly, Figure 5 presents the second difference in binding energy, calculated as

$$
\Delta_{2}(N)=2 E_{b}\left(\mathrm{Pt}_{N}^{A}\right)-E_{b}\left(\mathrm{Pt}_{N+1}^{A}\right)-E_{b}\left(\mathrm{Pt}_{N-1}^{A}\right) .
$$

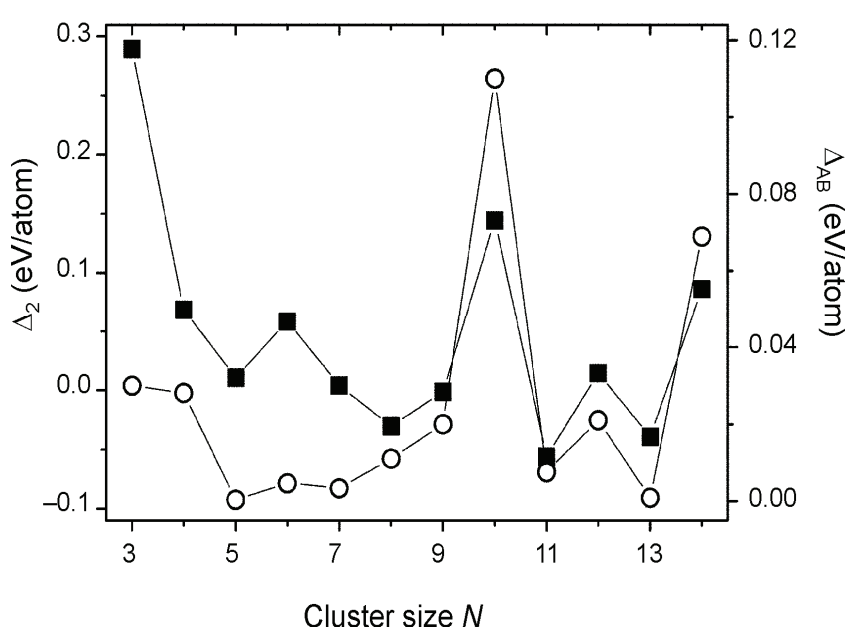

Fig. 5. $\Delta_{2}$ (filled squares) and $\Delta_{A B}$ (empty circles) as a function of cluster size

The energy distance to the second highly stable isomer, i.e.

$$
\Delta_{A B}(N)=E_{b}\left(\mathrm{Pt}_{N}^{A}\right)-E_{b}\left(\mathrm{Pt}_{N}^{B}\right),
$$

is also shown. Although both of these are somewhat oscillatory, they definitely indicate that $\mathrm{Pt}_{10}^{A}$ and $\mathrm{Pt}_{14}^{A}$ clusters demonstrate increased resistance to changes in size (high values of $\Delta_{2}$ ) and shape (high values of $\Delta_{A B}$ ). This leads us to conclude that during equilibrium-driven formation of clusters, a relatively higher occurrence of the isomers listed above may be observed. 
We found that the relative spacings between isomers (measured in the domain of binding energy) were significantly lower than those reported in [10] and [14]. This situation could be explained by the failure to account for spin-orbit effects in these works. Bhattacharyya and Majumder (who did not include these) found that the global minimum of the six-atom cluster prefers the geometry of stacked triangles (corresponding to $\mathrm{Pt}_{6}^{B}$ ), also concluding that the bi-capped rhombus isomer (equivalent to our $\mathrm{Pt}_{6}^{C}$ ) has a binding energy lower by $0.76 \mathrm{eV} /$ atom. Our investigations show that this difference is equal to $0.02 \mathrm{eV} /$ atom, which is almost forty times lower. Despite the fact that Xiao and Wang correctly recognized $\mathrm{Pt}_{6}^{A}$ and $\mathrm{Pt}_{6}^{B}$ to be almost isoenergetic, the GM of the six-atom cluster was found to possess a planar bi-capped square structure, which in our picture (see $\mathrm{Pt}_{6}^{J}$ ) displays extremely low stability. In addition, most of the structures which have been found by Xiao and Wang to be planar, in our case demonstrate geometries that are not planar but rather nearly-planar (bent). We stress that the above examples do not constitute exceptions and many other (even qualitative) differences are apparent between Xiao and Wang's and this work.

\section{SUMMARY}

Structural properties of small platinum clusters were studied at the DFT level of theory. Many distinct stable configurations were found during an exhaustive global search driven by a genetic algorithm. The obtained results show that up to $N=9$ planar and nearly planar arrangements are as stable as three dimensional ones. This trend turns into a tendency to prefer both distorted and disordered spatial structures beyond $N=9$. A detailed insight gained during this study shows that the global minima of $N=10$ - and 14-atom clusters have fcc-like structures and display increased stability. We thus conclude that even for the region of early growth, a relatively higher incidence of fcc-like structures should be experimentally observed, and not, as previously thought, icosahedral-like structures. A detailed comparison with results reported by other authors shows that accounting for spin-orbit coupling is crucial. Calculations that do not take this into account lead to PESs that display significantly larger energy barriers.

\section{Acknowledgements}

This work has been sponsored by the Ministry of Science and Higher Education, under a research grant number N N519 577838. The calculations were performed at the TASK Academic Computer Centre in Gdańsk, Poland.

\section{References}

[1] A. Capon, R. Parsons, J. Electroanal. Chem. 45, 205 (1973).

[2] J. Clavilier, R. Parsons, R. Durand, C. Lamy, J.M. Leger, J. Electroanal. Chem. 124, 321 (1981).

[3] H. Dahms, J.O.M. Bockris, J. Electrochem. Soc. 111, 728 (1964).

[4] S. Mukerjee, S. Srinivasan, J. Electroanal. Chem. 357, 201 (1993)

[5] K. Masaaki, I. Hirokazu, T. Masato, A. Masakazu, M.S-C, M. Hiroaki, S. Eiji, Y. Yuko, E. Takashi, Nippon Kagakkai Koen Yokoshu 81, 118 (2002).

[6] M. Takeuchi, S. Sakai, A. Ebrahimi, M. Matsuoka, M. Anpo, Topics in Catalysis 52, 1651 (2009).

[7] A. Sachdev, R.I. Masel, J. B. Adams, J. Catal. 136, 320 (1992).

[8] J.P.K. Doye, D.J. Wales, New J. Chem 733 (1998).

[9] S.H. Yang, D.A. Drabold, J.B. Adams, P. Ordejon, K. Glassford, J. Phys.: Condens. Matter 9, L39 (1997).

[10] N. Watari, S. Ohnishi, Phys. Rev. B 58, 1665 (1998).

[11] L. Xiao L. Wang, J. Phys. Chem. A 108, 8605 (2004).

[12] E. Apra, A. Fortunelli, J. Molecular Structure (Theochem) $501,251(2000)$.

[13] E. Apra, A. Fortunelli, J. Phys. Chem. A 107, 2934 (2003).

[14] A. Nie, J. Wu, C. Zhou, S. Yao, C. Luo, R.C. Forrey, H. Cheng, International Journal of Quantum Chemistry 107, 219 (2007).

[15] K. Bhattacharyya, C. Majumder, Chem. Phys. Lett. 446, 374 (2007).

[16] A. Sebetci, Phys. Chem. Chem. Phys. 11, 921 (2009).

[17] L.O. Paz-Borbon, Roy L. Johnston, A. Fortunelli, J. Phys. Chem. C 111, 2936 (2007).

[18] L.O. Paz-Borbon, A. Gupta, Roy L. Johnston, J. Mater. Chem. 18, 4154 (2008).

[19] L.O. Paz-Borbon, Roy L. Johnston, G. Barcaro, A. Fortunelli, J. Chem. Phys. 128, 134517 (2008).

[20] A. Longsdail, L.O. Paz-Borbon, Roy L. Johnston, Journal of Computational and Theoretical Nanoscience 6, 1 (2009).

[21] Roy L. Johnston, J. Chem. Soc. Dalton Trans. 4193 (2003).

[22] A.F. Voter, Los Alamos Unclassified Report LA-UR 93-3901 (1993).

[23] B. Hartke, J. Phys. Chem. 97, 9973 (1993).

[24] Y. Zeiri, Phy. Rev. E 51, R2769 (1995).

[25] D.M. Deaven, K.M. Ho, Phys. Rev. Lett. 75, 288 (1995).

[26] M.J. Frisch et al., GAUSSIAN 03, Revision E.01, Gaussian Inc., Wallingford, 2004.

[27] J.P. Perdew, Y. Wang, Phys. Rev. B 45, 13244 (1992).

[28] J.P. Perdew, J.A. Chevary, S.H. Vosko, K.A. Jackson, M.P. Pederson, D.J. Singh, C. Fiolhais, Phys. Rev. B 46, 6671 (1992).

[29] K.V. Tretiakov, K.W. Wojciechowski, Phys. Rev. E 60, 7626 (1999).

[30] A. Schafer, C. Huber, R. Ahlrichs, J. Chem. Phys. 100, 5829 (1994).

[31] D. Andrae, U. Haeussermann, M. Dolg, H. Stoll, H. Preuss, Theor. Chim. Acta 77, 123 (1990).

[32] B.M. Bode, M.S. Gordon, J. Mol. Graphics and Modeling $16,133(1998)$

[33] A. Marijnissen, T.T. ter Meulen, P.A. Hackett, B. Simard, Phys. Rev. A 52, 2606 (1995).

[34] N.D. Gibson, B.J. Davies, D.J. Larson, J. Chem. Phys. 98, 5104 (1993).

[35] M.M. Airola, M.D. Morse, J. Chem. Phys. 116, 1313 (2002).

[36] A. Grushow, K.M. Ervin, J. Chem. Phys. 106, 9580 (1997). 
SzYMon Winczewski was born in Koscierzyna, Poland in 1985. In 2009 he received his M.Sc. degree in Physics from Gdansk Univeristy of Technology. He is currently working on his doctoral dissertation which concerns the dislocation detection algorithms for atomistic simulations. His research interests include also the physics of nanosized systems.

JAROSŁAW RYBICKI is a professor in theoretical and computational physics in the Faculty of Applied Physics and Mathematics at the Gdansk University of Technology, Gdansk, Poland. His field of interest covers the structure of disordered systems (oxide glasses and liquid metals and alloys), phase transitions (condensation from gas phase, premelting phenomena), and the mechanical properties of nanostructures (mechanisms of plastic deformation, formation and motion of dislocations, molecular mechanisms of friction). Main research tools: classical and quatnum-classical simulations with particles, stochastic geometry methods. 